\title{
ina \\ Study on Influencing Factors of Helicopter Brownout Evolution Based on CFD-DEM
}

\author{
Yihua Cao, Gaozhan Wang * and Chongwen Jiang \\ School of Aeronautic Science and Engineering, Beihang University, Beijing 100191, China; \\ yihuacaobu@126.com (Y.C.); cwjiang@buaa.edu.cn (C.J.) \\ * Correspondence: wanggz0825@163.com
}

check for

updates

Citation: Cao, Y.; Wang, G.; Jiang, C.

Study on Influencing Factors of

Helicopter Brownout Evolution

Based on CFD-DEM. Appl. Sci. 2022,

12, 126. https://doi.org/10.3390/

app12010126

Academic Editors: Roman Starosta,

Jan Awrejcewicz, José A.

Tenreiro Machado, José M. Vega, Hari

Mohan Srivastava, Ying-Cheng Lai

and Hamed Farokhi

Received: 3 November 2021

Accepted: 20 December 2021

Published: 23 December 2021

Publisher's Note: MDPI stays neutral with regard to jurisdictional claims in published maps and institutional affiliations.

Copyright: (c) 2021 by the authors Licensee MDPI, Basel, Switzerland. This article is an open access article distributed under the terms and conditions of the Creative Commons Attribution (CC BY) license (https:// creativecommons.org/licenses/by/ $4.0 /)$.

\begin{abstract}
The gas-solid two-phase flow model is constructed based on the Euler-Lagrangian framework. The SST $k-\omega$ two-equation turbulence model and the soft ball model are coupled by computational fluid dynamics (CFD) and a discrete element model (DEM). Brownout is then simulated by the above method with sliding mesh. As the calculation examples show, the simulations and experiments of the Lynx rotor and the Caradonna-Tung rotor are compared. The coupling method is verified through calculation of the rotor lift coefficient, blade section pressure coefficient and tip vortex shedding position. The results show that when the helicopter is hovering at a height of $0.52 \mathrm{R}$ from the ground, it will cause brownout and the pilot's vision will be obscured by sand. When the hovering height is $1 \mathrm{R}$, the phenomenon of brownout is not serious. The movement speed of most sand dust is about $12 \mathrm{~m} / \mathrm{s}$, and the height of the sand dust from the ground will gradually increase over time. Large particles of sand are more difficult to be entrained into the air than the small particles, and the particles with a radius of $50 \mathrm{um}$ are basically accumulated on the ground. Moreover, the slotted-Tip rotor has an effect on restraining brownout.
\end{abstract}

Keywords: helicopter; brownout; computational fluid dynamics; discrete element; gas-solid twophase flow

\section{Introduction}

Brownout is the name of the limited visual field of a helicopter pilot after environmental impact, which easily occurs in a sand-dust environment. The main influence area of brownout is the circle around the helicopter which is several times of the radius of the rotor. The hovering flow field will entrain many sandy particles from the ground into the air, thus reducing the pilot's visibility of the surrounding environment. The degree of visual limitation depends on the type of helicopter (single rotor or coaxial twin-rotor), and the operation condition (helicopter landing posture or flight trajectory). In this environment, the pilot can easily lose a sense of space and direction, and can become unable to judge the ground position, horizontal attitude and other information accurately. The pilot will then make mistakes in the control of the helicopter, which can cause the helicopter to crash and result in injury to the pilot.

As early as the 1940s, Bagnold [1] started to study the problem of wind-drift sand in the desert of Libya. Combined with wind tunnel experiments, he produced a series of formulas for the efficiency of wind-drift sand transportation. Wachspress et al. [2] established a dusty entrainment model using Bagnold's method. In the 1960s, Sutherland [3] studied the way that turbulence carries sand from the ground. An entrainment hypothesis that turbulent eddy currents destroy the viscous layer and directly impact on the sand surface was proposed. Leese and others of the US Army [4] studied the entrainment of the downwash flow when the helicopter is near the ground, which is called the blast effect. Subsequently, Rodgers [5] carried out several large-scale experimental studies using a twinrotor helicopter which hovered near the ground with a dust collector. As a result, it was found that the entrainment particles are larger when the helicopter is closer to the ground. 
In the 21st century, particle image velocimetry (PIV) have become the mainstream methods for researchers to analyze brownout. Leishman et al. [6] used PIV to study the two-phase flow environment caused by a helicopter hovering on sands. It was found that in the process of the viscous merging of an adjacent wake vortex, the velocity of the upward flow increases significantly. It was observed that the sandy particles with enough height will recycle into the rotor wake and spray to the ground, causing dusty entrainment through the process of bombardment. Lee et al. [7] conducted experiments based on PIV and found that the viscous diffusion process, strain-induced vorticity enhancement process and the generations of turbulence are the factors that affect the flow field characteristics. Nathan and Green [8] used PIV to observe the flow structure in front of and under the helicopter rotor during forward flight and found that forward flight has a significant effect on the wake compared with hovering, and the rotor wake will be closer to the blade and smaller.

Ramasamy and Leishman [9] studied the effects of vortex filament deformation and viscous diffusion. Syal and Leishman [10] used the Lagrangian method to calculate the movement of particles in the flow field, and simulated the two-phase flow containing sand. Milluzzo et al. [11] used PIV to study the scaled model with four different tip shapes, and measured the vortex core size and the vortex sheet velocity at $800^{\circ}$ azimuth. The results show that there are some common flow characteristics among the four different tip shapes, and the difference of flow may ultimately affect the evolution of brownout. The slotted tip is especially effective in diffusing the tip vortex.

Polzin et al. [12] used a computational fluid dynamics (CFD) method to study the influence of a discrete rotor blade model in the dusty cloud caused by helicopter downwash. The results of the wake velocity profile, ground shear force profile and dusty cloud density at different heights of the independent rotor in hover are given and compared with the steady model. Hance et al. [13] added fuselage to the simulation to study the influence of the fuselage on rotor flow field in the brownout phenomenon.

Whitehouse et al. [14] conducted brownout experiments using a scaled model (chord length of 1 inch, tip velocity of $200 \mathrm{ft} / \mathrm{s}$ ) with the same strength as the full-scale helicopter tip vortex. It was found that the dissipation of the tip vortex can be accelerated by adding a winglet at the trailing edge of the blade. Subsequently, they [15] established the key relationship between the rotor wake and tip vortex characteristics in the brownout phenomenon and developed a method to reduce the entrainment effect of the brownout phenomenon without changing the aerodynamic performance of the rotor.

Taking UH60 as an example, we study the factors affecting brownout during hovering, which can provide suggestions to pilots flying in relevant environments. We analyze a kind of blade structure, and discuss its effect on suppressing brownout.

\section{Numerical Simulation Theory}

\subsection{Governing Equation}

In computational fluid dynamics, the computer obtains the physical parameters of the flow field by solving the governing equations, which are generally considered as the mass conservation equation, momentum conservation equation and energy conservation equation [16].

The mass conservation equation is also called a continuity equation. According to the law of mass conservation, the mass of fluid passing through the surface of volume in the flow field is consistent with the variable controlling the mass of fluid in the volume.

$$
\oiint_{C S} \rho v_{n} d A+\frac{\partial}{\partial t} \iiint_{C V} \rho d V=0
$$


where $\rho$ is the density of the fluid, $v_{n}$ is the normal velocity, $A$ is the surface area of the control volume, and $V$ is the volume The continuity equation of any point in the flow field can be deduced by using the above integral form of mass conservation equation:

$$
\frac{\partial \rho}{\partial t}+\operatorname{div}(\rho \vec{v})=0
$$

This is the continuous differential equation of unsteady compressible flow.

For steady flow:

$$
\frac{\partial \rho}{\partial t}=0
$$

For incompressible flow, $\rho$ is a constant

$$
\operatorname{div}(\vec{v})=\frac{\partial v_{x}}{\partial x}+\frac{\partial v_{y}}{y}+\frac{\partial v_{z}}{z}=0
$$

In the following cases, the rotor tip Mach number is much less than 1, and it could be approximately considered as an unsteady incompressible flow.

A momentum conservation equation, known as a Navier-Stokes (N-S) equation, refers to the change rate of fluid momentum relative to time in the fluid micro cluster, which is the sum of external forces on the fluid micro cluster. The formula is as follows:

$$
\frac{\partial \rho \vec{U}}{\partial t}+\operatorname{div}(\rho \vec{U} \vec{U})=\rho \vec{f}-\operatorname{grad}(p)+\operatorname{div}(\vec{\tau})
$$

where $\vec{f}$ is the volume force, $\rho \vec{U}$ is the mass flow rate, $p$ is the pressure, and $\vec{\tau}$ is the shear stress tensor.

For incompressible fluid, the above formula can be simplified and expressed as follows:

$$
\begin{aligned}
& \frac{d v_{x}}{d t}=f_{x}-\frac{1}{\rho} \frac{\partial p}{\partial x}+v\left(\frac{\partial^{2} v_{x}}{\partial x^{2}}+\frac{\partial^{2} v_{x}}{\partial y^{2}}+\frac{\partial^{2} v_{x}}{\partial z^{2}}\right) \\
& \frac{d v_{y}}{d t}=f_{y}-\frac{1}{\rho} \frac{\partial p}{\partial y}+v\left(\frac{\partial^{2} v_{y}}{\partial x^{2}}+\frac{\partial^{2} v_{y}}{\partial y^{2}}+\frac{\partial^{2} v_{y}}{\partial z^{2}}\right) \\
& \frac{d v_{z}}{d t}=f_{z}-\frac{1}{\rho} \frac{\partial p}{\partial z}+v\left(\frac{\partial^{2} v_{z}}{\partial x^{2}}+\frac{\partial^{2} v_{z}}{\partial y^{2}}+\frac{\partial^{2} v_{z}}{\partial z^{2}}\right)
\end{aligned}
$$

where the kinematic viscosity $v=\mu / \rho$ is the ratio of dynamic viscosity to density.

The above are the governing equations of continuous medium hydrodynamics. The computer divides the flow field into a large number of grids based on the finite volume method and solves them. In some cases, it is necessary to add other equations for closure.

\subsection{Turbulence Model}

Turbulence is an inevitable problem in the simulation of flow field. The rotor flow field of helicopter is a highly nonlinear turbulent flow, so before conducting the numerical simulation of a rotor flow field, it is necessary to study the turbulence model and add an appropriate turbulence model in the flow-governing equation to obtain a reasonable solution and complete the flow field simulation.

The SST $k-\omega$ two-equation model uses the $k-\omega$ model in the boundary layer, which has better treatment effect in the viscous sublayer region. It is transformed into a $k-\varepsilon$ model in the outer layer, which avoids the problem that the $k-\omega$ model is too sensitive to the turbulent characteristics. The viscous formula is modified by considering the shear stress in the turbulent flow. It is more suitable for high curvature flow, flow with obvious 
separation and jet flow, and more accurate for the rotor flow field model. The turbulent kinetic energy $k$, turbulent dissipation rate $\varepsilon$ and specific dissipation rate $\omega$ are as follows:

$$
\begin{gathered}
k=\frac{\overline{u_{i}^{\prime} u_{i}^{\prime}}}{2} \\
\varepsilon=\frac{\mu}{\rho} \overline{\left(\frac{\partial u_{i}^{\prime}}{\partial x_{k}}\right)\left(\frac{\partial u_{i}^{\prime}}{\partial x_{k}}\right)} \\
\omega=\frac{\varepsilon}{k}
\end{gathered}
$$

\subsection{Gas-Solid Two-Phase Flow Model}

For the description of the brownout phenomenon, the most appropriate model is the gas-solid two-phase flow model. In the simulation of the gas-solid two-phase flow, the force between fluid and solid, and the force between solid particles and the wall should be considered. At present, the research of the gas-solid two-phase flow has been more mature, and the main models used are the Euler-Euler method, Euler-Lagrange method, and the dynamic model of particle impingement.

The Euler-Lagrange method is usually used when considering the motion of a single particle or particles. The fluid phase is still described by the Euler method, and the particle motion is described by ordinary differential equations in Lagrange coordinates.

In recent years, a discrete element model (DEM) considering particle collision has been developed, and this can be applied to a dense gas-solid two-phase flow. In addition, the model does not directly solve the flow field around the particles and uses a suitable drag model to replace the force between the particles and flow field, which greatly reduces the calculation cost and can track a high number of particles.

When calculating the force between particles, it is necessary to consider the collision of particles. The common models are the soft ball model and the hard ball model. In this paper, the soft ball model is adopted. The basic assumption is that the particle collision will last for some time, and there will be slight deformation in the collision process (which is shown as overlapping phenomenon in the calculation). On this basis, the spring and damper are used to simulate the particle collision phenomenon.

The hard sphere model is based on the law of impulse conservation, and the basic assumption is that the collision process of particles is completed in an instant without considering the deformation of particles. In the mathematical model, the collision is completed in a time step, and the recovery coefficient is used to solve the velocity after collision.

The specific process of simulation is to calculate the kinetic energy loss through the material characteristics and the particle velocity at the time of collision, and to reflect the kinetic energy loss by applying a force in the opposite direction to the particle. The normal deformation and tangential deformation of particles are then calculated according to the material rigidity, particle velocity and resistance. At the same time, the normal resistance and tangential resistance are calculated from the deformation and kinetic energy loss. The normal force will make the particles rebound, and the tangential force will make the particles rotate.

The gas-solid two-phase flow model's calculation process is shown in Figure 1: 


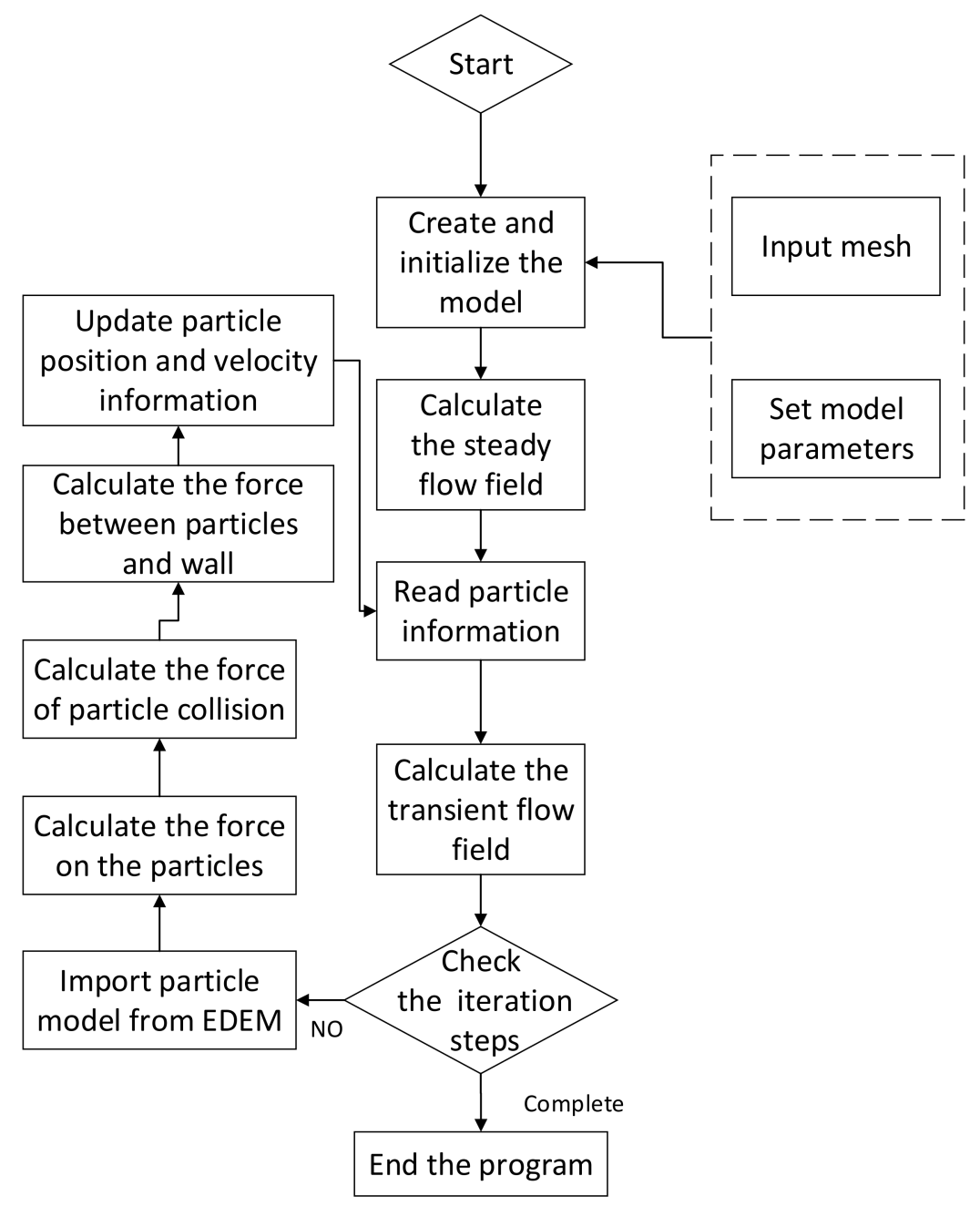

Figure 1. Calculation process.

\section{Verification of Calculation Method}

A full scale Lynx rotor and Caradonna-Tung rotor with detailed experimental results were selected as verification cases [17-19]; the simulation variables are the hovering height, pitch angle and tip Mach number. The simulation results are compared with the experimental results by lift coefficient and surface pressure coefficient to prove the reliability of the numerical method.

\subsection{Lynx Rotor}

In this experiment, the inflow velocity is $2.5 \mathrm{~m} / \mathrm{s}$ and the tip Mach number is 0.60 . The geometric parameters are shown in Table 1 . The blade plane reference diagram is shown in Figure 2.

Table 1. Geometric parameters.

\begin{tabular}{ccc}
\hline Airfoil & Disk Radius (mm) & Chord Length (mm) \\
\hline NPL9615 & 1105 & 180 \\
\hline
\end{tabular}




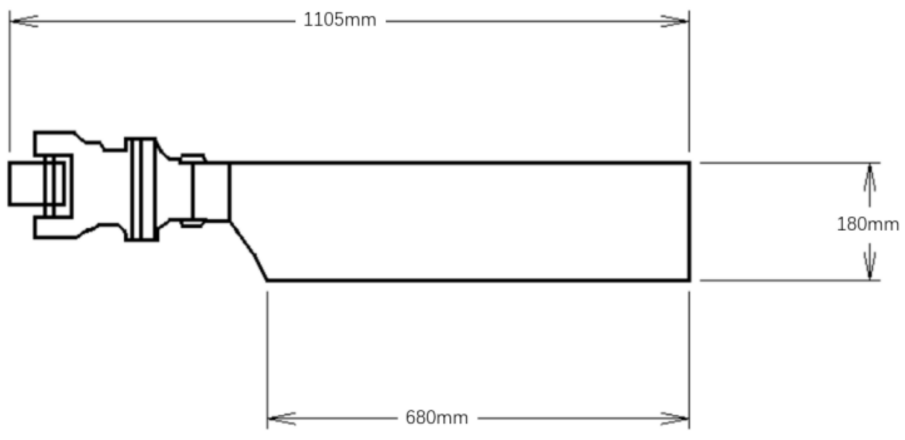

Figure 2. Lynx tail rotor characteristics.

Five groups of data are selected for comparative verification. The lift values obtained by simulation are shown in Table 2. The values are compared with the experimental results through the form of the lift coefficient, as shown in Figure 3.

Table 2. Simulation parameters.

\begin{tabular}{cccc}
\hline Number & Hover Height & Pitch $\left(^{\circ}\right)$ & Lift (N) \\
\hline 1 & $0.84 \mathrm{R}$ & 13 & 2871.36 \\
2 & $0.64 \mathrm{R}$ & 14 & 3486.65 \\
3 & $0.52 \mathrm{R}$ & 15 & 3933.99 \\
4 & $0.72 \mathrm{R}$ & 16 & 4078.84 \\
5 & $0.78 \mathrm{R}$ & 17 & 4340.15 \\
\hline
\end{tabular}

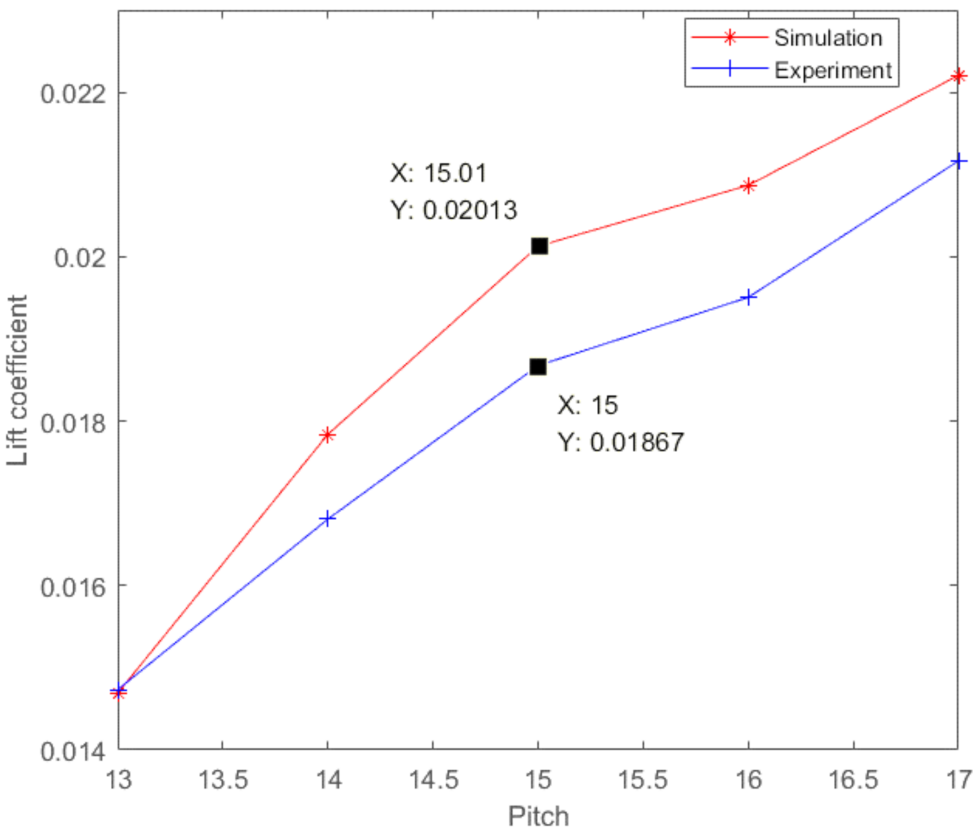

Figure 3. Comparison of lift coefficient results.

From the above figures, the rotor lift is positively correlated with the collective pitch degree. Comparing the simulation and experimental results, the data error of the lift coefficient is less than $8 \%$ under different working conditions. The validation of the rotor lift coefficient in different cases shows that the grid and turbulence model used in this paper are reliable. 


\section{2. "Caradonna \& Tung" Rotor}

Four groups of experiments were simulated and the surface pressure coefficient of rotor was compared. The geometric and simulation parameters are shown in Tables 3 and 4.

Table 3. Geometric parameters.

\begin{tabular}{ccc}
\hline Airfoil & Disk Radius $(\mathbf{m m})$ & Chord Length $(\mathbf{m m})$ \\
\hline NACA0012 & 1143 & 190.5 \\
\hline
\end{tabular}

Table 4. Simulation parameters.

\begin{tabular}{ccc}
\hline Number & Tip Mach Number & Pitch $\left(^{\circ}\right)$ \\
\hline 1 & 0.439 & 8 \\
2 & 0.612 & 8 \\
3 & 0.794 & 8 \\
4 & 0.877 & 8 \\
\hline
\end{tabular}

Select the section with $\mathrm{r} / \mathrm{R}=0.8$ on the blade for calculating the surface pressure coefficient. The results are shown in Figures 4 and 5.
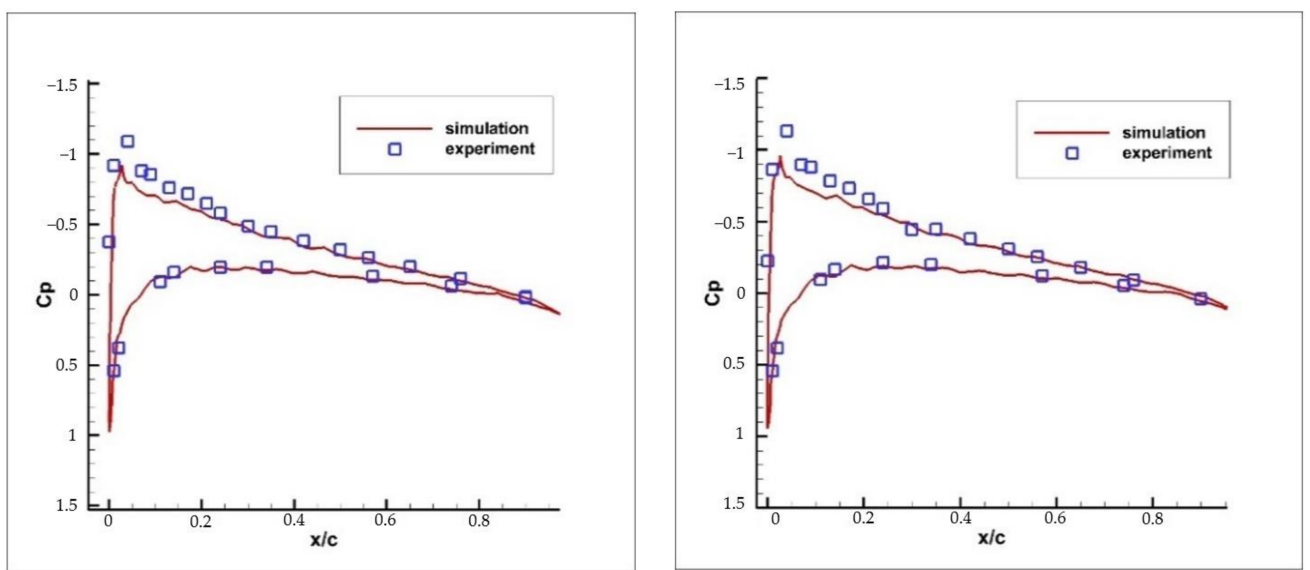

Figure 4. Group 1 Results and Group 2 Results.
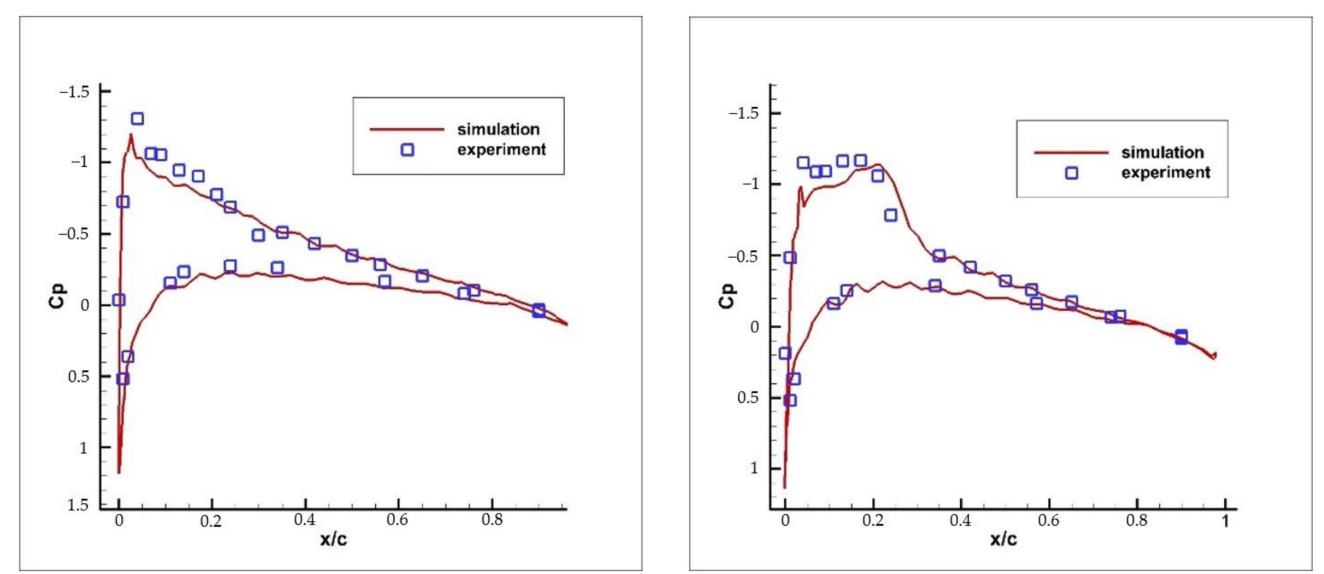

Figure 5. Group 3 Results and Group 4 Results.

From the above figures, the overall trend of the pressure coefficient calculated by the simulation is consistent with the experimental results at different Mach numbers; the data 
are almost consistent but errors exist at the leading edge of the airfoil, which ranges from $15 \%$ to $20 \%$. When the Mach number reaches a certain value, the position of the maximum pressure coefficient of the airfoil will move backward from the leading edge of the airfoil.

\section{Coupling Simulation Analysis}

The above two blades prove the reliability of the simulation model, but due to the lack of corresponding fuselage, the classic helicopter UH60 was selected to analysis the brownout. The discrete element model and computational fluid dynamics model were used for coupling calculation to analyze the formation and evolution of brownout. The software used are EDEM and FLUENT. The fluid solver is based on RANS with the SST $k-\omega$ turbulence model. The spatial discretization is second order upwind for momentum, turbulent kinetic energy and specific dissipation rate.

The UH60 adopts three-stage twist and swept rotor blades, with the airfoil of SC1095 and SC1094R8. The tip Mach number is 0.63 , the collective pitch is $9^{\circ}$, the blade radius is $8200 \mathrm{~mm}$, chord length is $530 \mathrm{~mm}$, the tip swept angle is $20^{\circ}$ and the negative twist angle is $13^{\circ}$ [20]. The model uses an unstructured grid with a total of 12 million. The time step size is the time taken for one degree per revolution of the blade tip.

When the height is $h / R=0.52$, the rotor ground effect flow field is calculated by using the sliding grid method. The time step size is the time required for each degree of blade rotation. The velocity and streamline distribution are shown in Figures 6 and 7. The $x$ direction is the in-coming flow direction, and the $\mathrm{z}$ direction is the direction perpendicular to the ground.

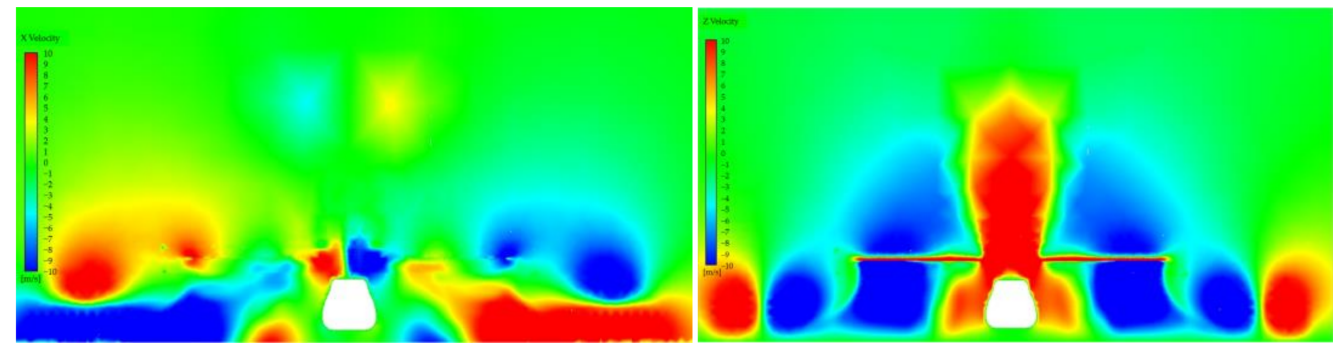

Figure 6. X-direction and Z-direction velocity contours.

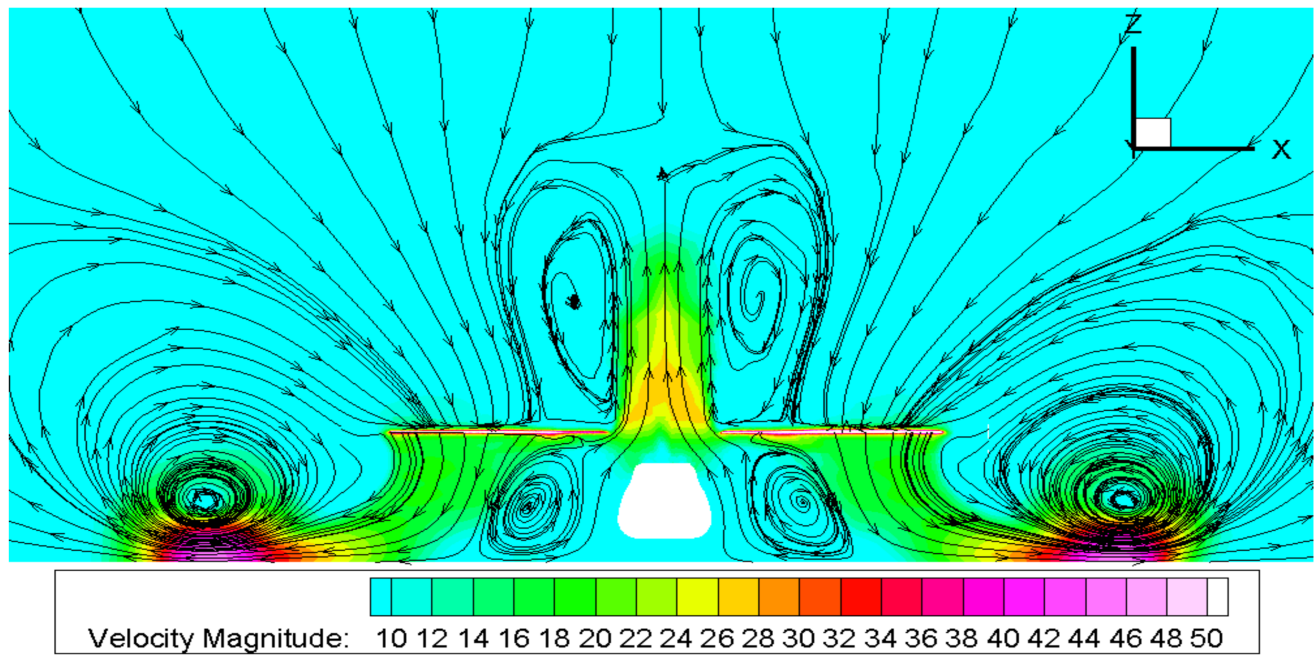

Figure 7. Section velocity contours and streamline distribution.

From the above figure it can be seen that there is obvious swirling air flows under the out-ward side of the blade, and that the air flows down from the tip of the blade and rolls 
back over the blade when it reaches the ground. There are also two smaller raceways above the blade root, which are judged to be caused by the high-intensity vortex.

In the process of the coupling simulation, the particles are simplified as spherical particles in the sand model, and the interaction between particles is solved by the Hertz Mindlin (no slip) model, and the interaction between particles and wall (including fuselage, blade and ground) is solved by the Hertz Mindlin with JKR (Johnson Kendall Roberts) contact model. The particles and the flow field exhibit two-way coupling. The flow field is solved for $0.5 \mathrm{~s}$ to achieve stability, then 5000 to 20,000 particles are added in the vicinity of the ground according to the time for calculation. The physical parameters of the particles are shown in Table 5 [21].

Table 5. Particle physical parameters.

\begin{tabular}{|c|c|c|c|c|c|}
\hline $\begin{array}{l}\text { Density } \\
\left(\mathrm{kg} / \mathrm{m}^{3}\right)\end{array}$ & $\begin{array}{l}\text { Poisson's } \\
\text { Ratio }\end{array}$ & $\begin{array}{l}\text { Shear } \\
\text { Modulus }\end{array}$ & $\begin{array}{l}\text { Coefficient of } \\
\text { Restitution }\end{array}$ & $\begin{array}{l}\text { Static Friction } \\
\text { Coefficient }\end{array}$ & $\begin{array}{l}\text { Rolling Friction } \\
\text { Coefficient }\end{array}$ \\
\hline 2710 & 0.25 & $1 \times 10^{8}$ & 0.5 & 0.4 & 0.05 \\
\hline
\end{tabular}

\subsection{Result of Hovering Height $0.52 R$}

The movement and distribution of simulated sand particles are studied. Figures 8-10 show the distribution of sand around the fuselage at different times when the hovering height of the UH60 is $0.52 \mathrm{R}$, the particle radius is $10 \mathrm{um}$, and the number of increased particles is 2500 per second.

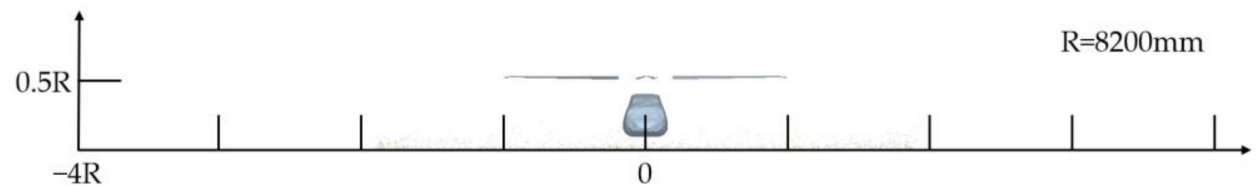

Figure 8. Dust distribution at $1 \mathrm{~s}$.

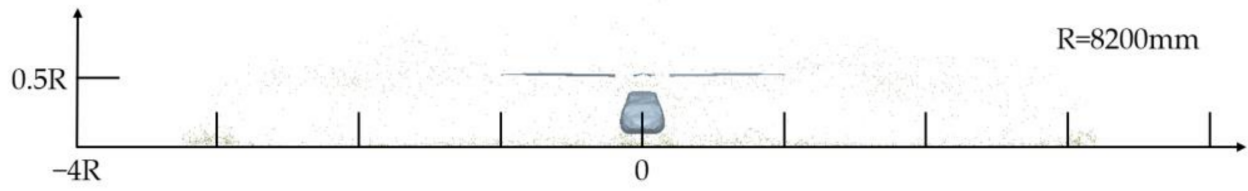

Figure 9. Dust distribution at $2 \mathrm{~s}$.

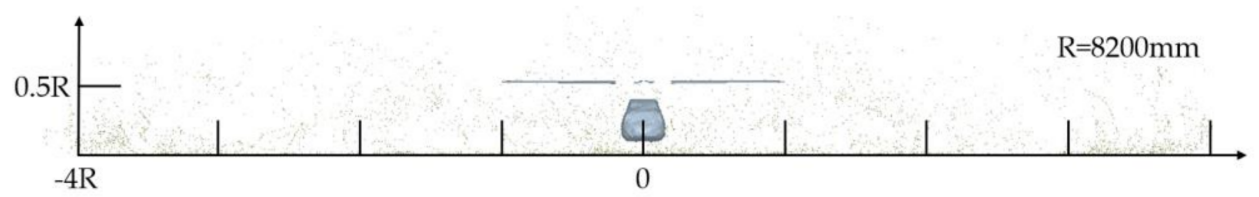

Figure 10. Dust distribution at $3 \mathrm{~s}$.

It can be seen from the above figure that, in a short time, the sand particles will be entrained into the air. Some particles will be suspended in the air under the influence of the ground eddy current, and will then contact the blade disk again to enter the downwash air flow cycle. A small number of particles will be suspended above the blade disk. In the following time, the sands mainly move in the spanwise direction, and the expansion speed gradually slows down. The sandy cloud has no significant change of height in the radial direction, which indicates that the main accumulation area of the dusty cloud is around this hovering height shown in Figure 10. The particles under the fuselage will be carried to a higher position by the rising air over time. 
In the brownout phenomenon, the height of sand particles is related to the probability of dust collision with the fuselage or blade, and to the degree of wear and erosion of the blade surface. The height is also related to the visual field of the pilot in the cockpit. The height of particles from $0 \mathrm{~mm}$ to $4000 \mathrm{~mm}$ is counted; after this, every $200 \mathrm{~mm}$ is the interval. The particles leaving the ground $(\mathrm{d}>200 \mathrm{~mm})$ are analyzed separately, and the average height is counted. The results are shown in Figures 11 and 12.
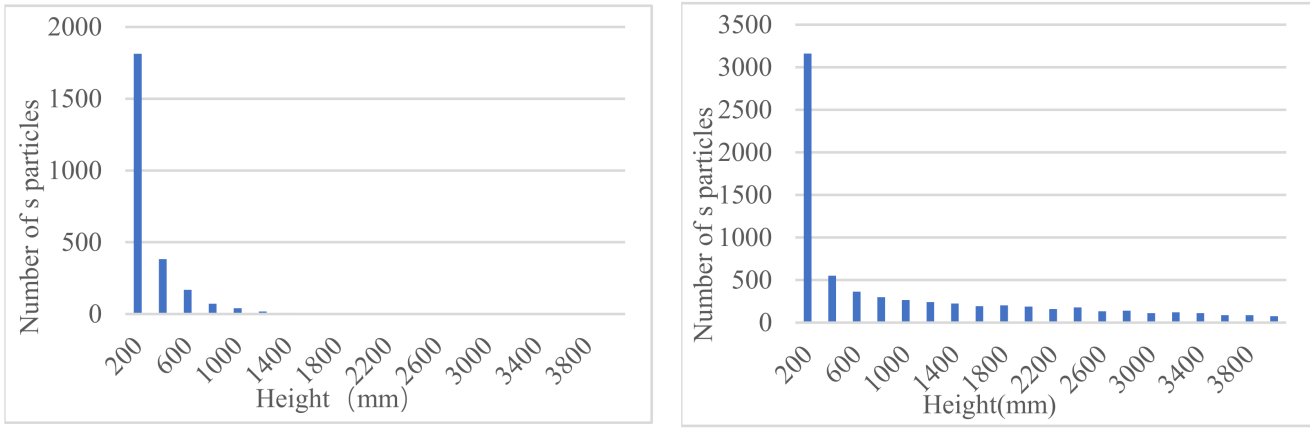

Figure 11. The number of particles at $1 \mathrm{~s}$ and $3 \mathrm{~s}$.
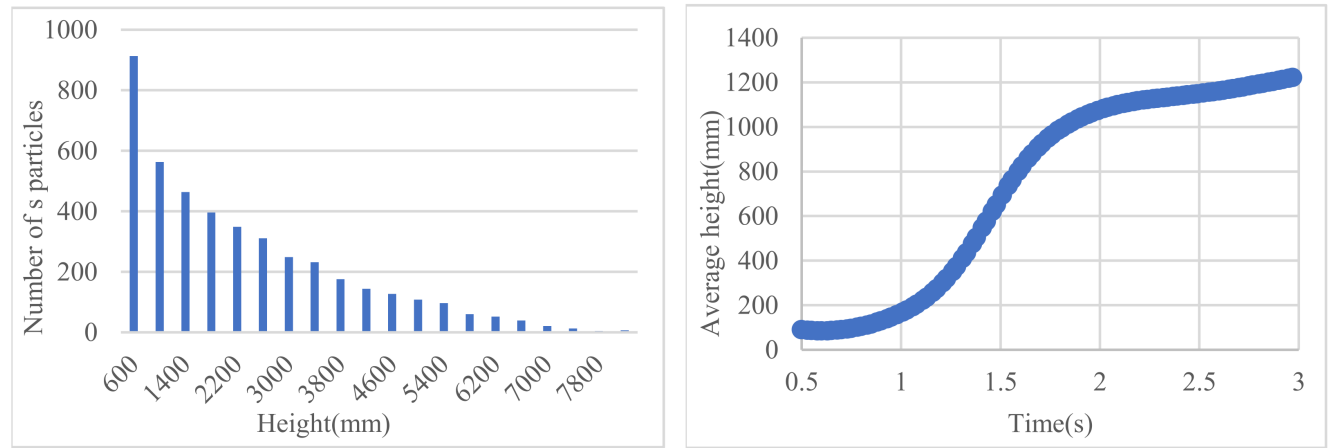

Figure 12. The number of suspended particles at $3 \mathrm{~s}$ and the average height of particles in $3 \mathrm{~s}$.

From Figure 11, it can be seen that the height of particles from the ground increases gradually within $3 \mathrm{~s}$, but more than $50 \%$ of the particles are still concentrated near the ground $(0-0.1 \mathrm{R})$ and are not entrained into the air by the airflow. The height of the other particles increases gradually in $3 \mathrm{~s}$, and the vertical distribution is more uniform.

From Figure 12 it can be seen that, when $t=3 \mathrm{~s}$, the number of suspended particles starts from $200 \mathrm{~mm}$ above the ground, and the number of suspended particles is negatively correlated with the height above the ground, and gradually decreases. The higher part has exceeded the height of $1 \mathrm{R}$, while the particles near the position of $0.5 \mathrm{R}(3800 \mathrm{~mm}-4600 \mathrm{~mm})$ account for about $2 \%$. This part of particles may cause erosion to the blades. Particles near the cockpit height of $0.3 \mathrm{R}(2200 \mathrm{~mm}-3000 \mathrm{~mm})$ account for about 5\%, which will affect the pilot's vision. The average height of particles shows a rapid upward trend between $1 \mathrm{~s}$ to 2 $\mathrm{s}$, and the average height gradually flattens after $2 \mathrm{~s}$.

In addition to the height, the movement speed of sand particles is also the basis for judging the brownout phenomenon, and the movement speed is related to the severity of sand erosion on the fuselage and blades. The distribution of particle velocity and the relationship between the average velocity and time are shown in Figures 13 and 14 . 

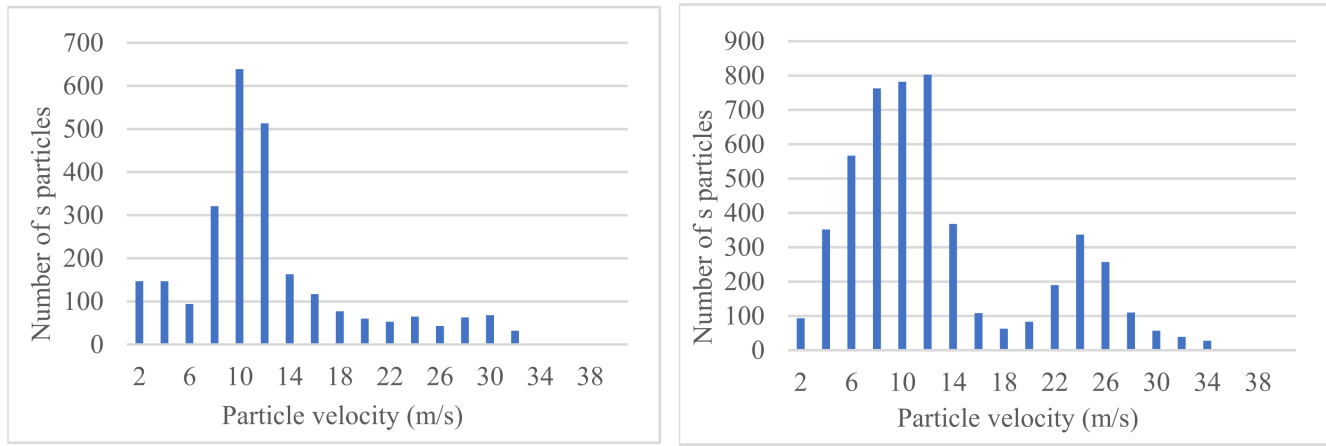

Figure 13. Particle velocity distribution at $1 \mathrm{~s}$ and $2 \mathrm{~s}$.
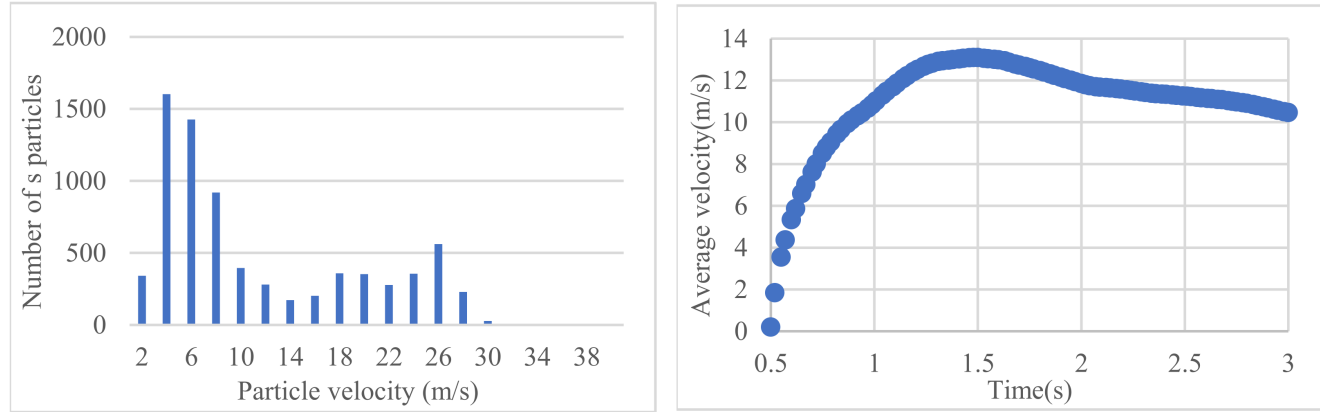

Figure 14. Particle velocity distribution at $3 \mathrm{~s}$ and the average velocity of particles in $3 \mathrm{~s}$.

Just after the particles are entrained off the ground $(t=1 \mathrm{~s})$, the velocity is mainly concentrated between $10 \mathrm{~m} / \mathrm{s}$ and $14 \mathrm{~m} / \mathrm{s}$. The number of particles moving at a high speed is less, and the overall average velocity is not fast. With the passage of time, the average velocity of particles gradually increases. After $2 \mathrm{~s}$, the number of high-speed particles gradually increases, and the number of particles with a velocity of about $25 \mathrm{~m} / \mathrm{s}$ also increases, while the average velocity of the particles with the largest proportion begins to decrease. When $t=3 \mathrm{~s}$, the trend is more obvious; some particles have a longer time to experience the acceleration of air flow, the proportion of particles with high-speed motion increases significantly, and the other particles will be affected by the air flow. The velocity begins to decrease after the particles suspend or sink to the bottom.

\subsection{Results of Hovering Height $1 R$}

When considering the influence of dust in the rotor flow field, the ground effect will have a great influence on the formation process of brownout, and the hovering height is the decisive factor of the ground effect. Therefore, changing the hovering height to observe the movement and distribution of dust is the research focus. The following is the calculation results when the hovering height is $1 R$, in which the number of dust growth is 5000 per second. The results are shown in Figures 15 and 16.

Compared with the hovering height of $0.52 R$, when the height from the ground is $1 R$, most of the particles move near the ground in $4 \mathrm{~s}(\mathrm{H}<250 \mathrm{~mm})$, but the overall average height of particles will gradually increase with time, and the proportion of particles leaving the ground is also increasing. 
Apple. Sci. 2022, 12, 126

12 of 16
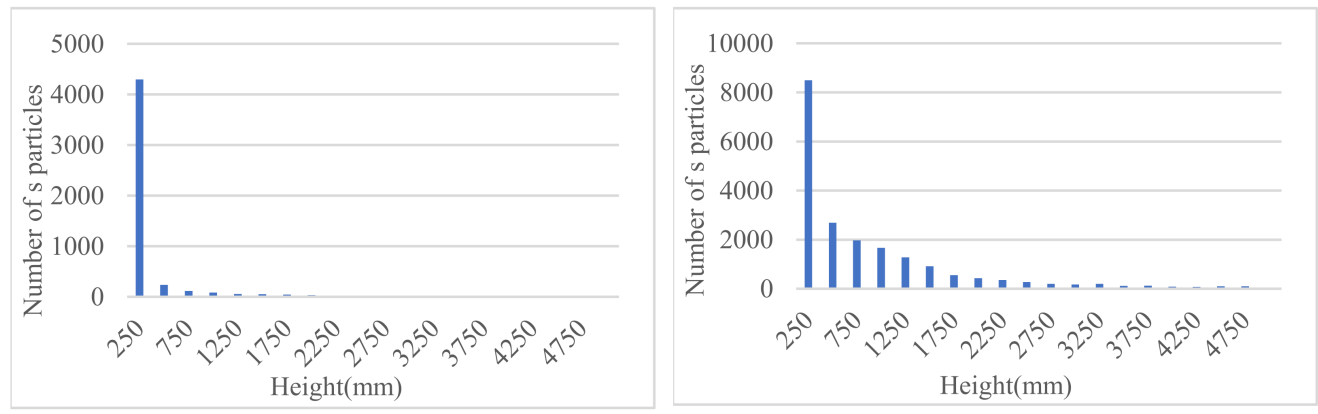

Figure 15. The number of particles by height at $1 \mathrm{~s}$ and $4 \mathrm{~s}$.
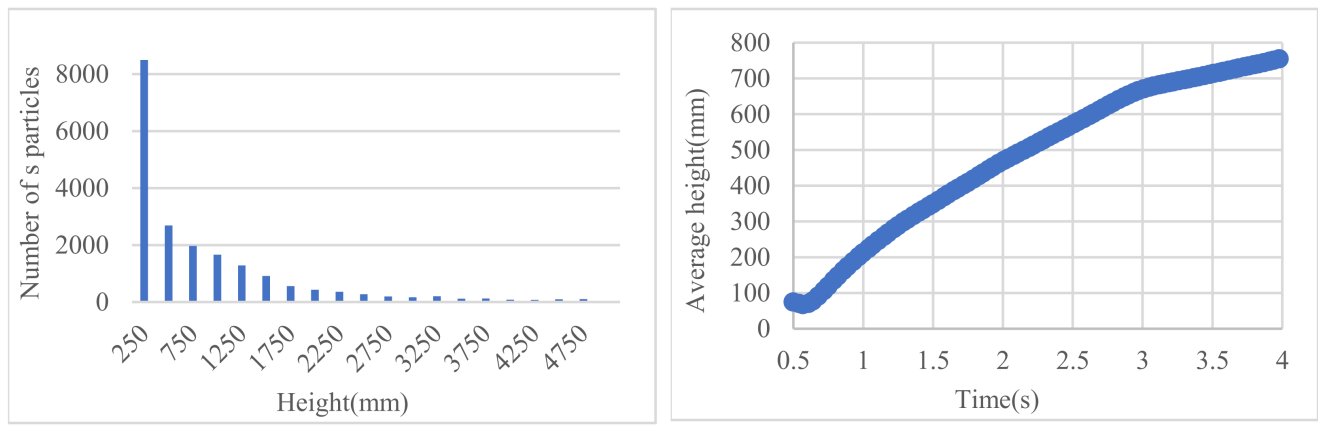

Figure 16. The number of particles by height at $4 \mathrm{~s}$ and the average height of particles in $4 \mathrm{~s}$.

4.3. Influence of Sand Particle Size

The size of sand particles will affect the force of dust in the flow field, and then affect the movement and distribution of dust. Considering that there are various particles of different sizes in the actual sand-dust environment, this paper compares the dust particle radius of $10 \mathrm{um}$ and $50 \mathrm{um}$, and the results are shown in Figures 17-19.

The above Figure indicates that the sand particles with a radius of 50 um basically stay on the ground and a small part of them are suspended in the air. The particles staying on the ground will move with the air flow like 10 um particles, while $10 \mathrm{um}$ particles will be suspended in the air. However, there is little difference in the velocity between the two types, which indicates that the radial motion affects the overall velocity.

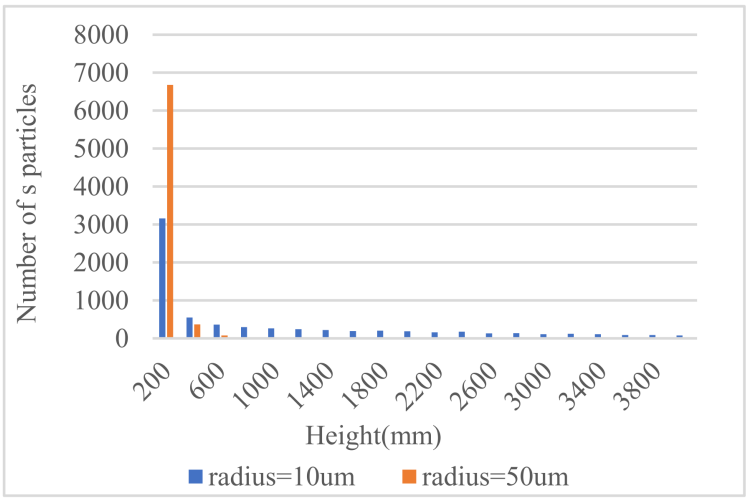

Figure 17. The number of particles by height at $3 \mathrm{~s}$. 

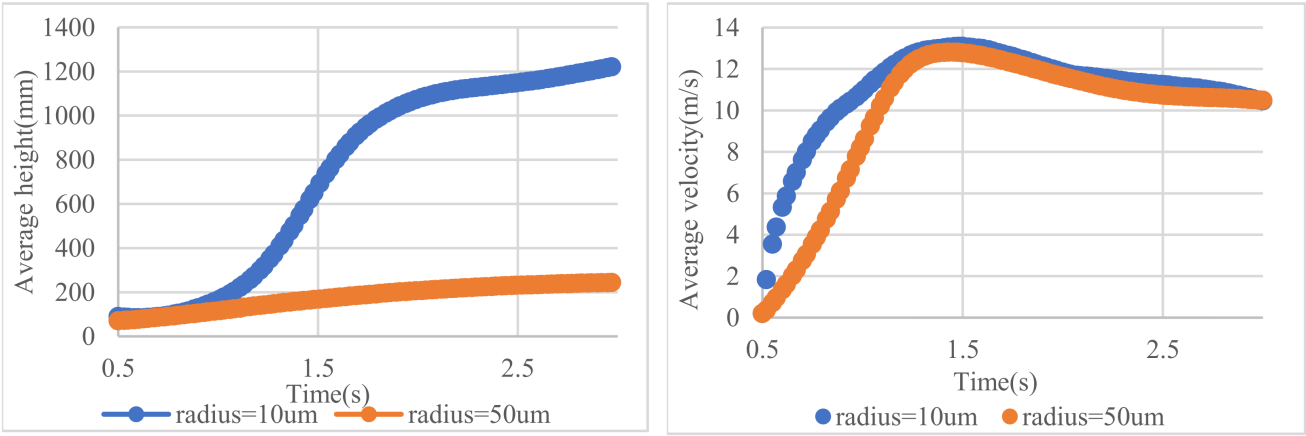

Figure 18. The average height of particles in $4 \mathrm{~s}$ and the average velocity of particles in $3 \mathrm{~s}$.

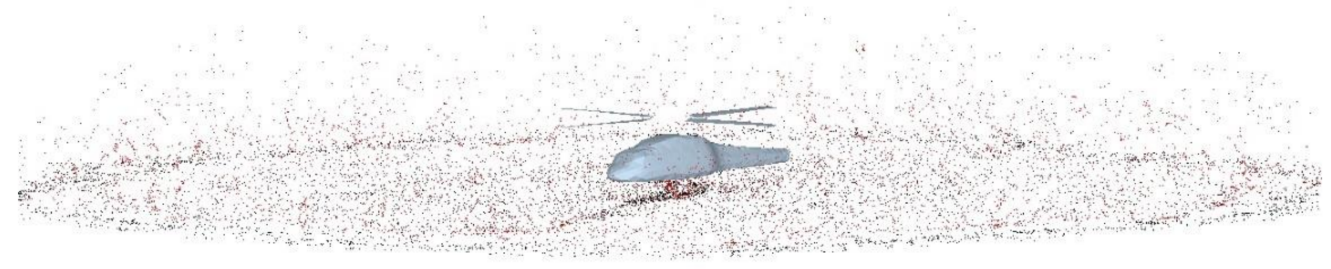

Figure 19. Distribution of sand particles.

\subsection{Effect of Slotted-Tip in Brownout}

By slotting the blade tip and connecting the leading edge and side of the blade tip with a socket, the blade vortex interaction (BVI) noise of the blade can be effectively reduced. The principle is that the leading-edge slot guides the airflow to the side flow around the blade tip and flows in the core area of the blade tip vortex to form part of the turbulent region, which destroys the original layout of vortex's high-speed rotating flow to inhibit the formation of turbulence in the blade tip region, also speeds up the dissipation of the blade tip vortex, and finally weakens the strength of the blade tip vortex.

The blade of the UH60 is improved by using this method. Four circular slots are added at the blade tip. The model is shown in Figure 20.

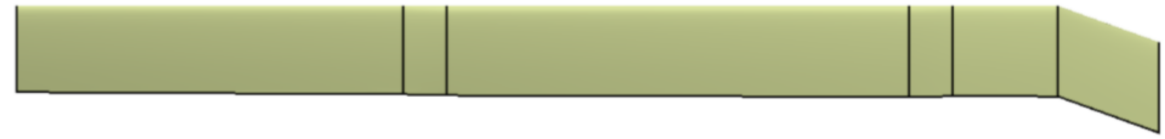

(a)

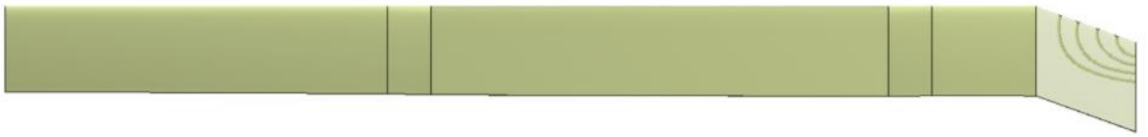

(b)

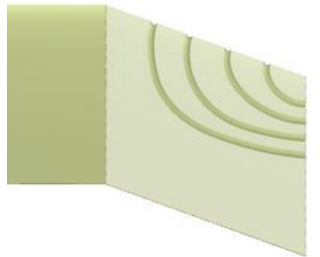

(c)

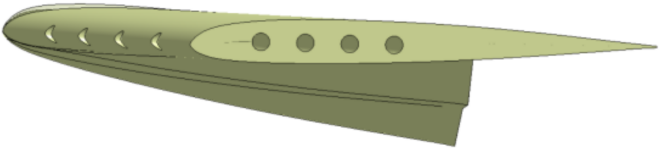

(d)

Figure 20. Slotted-tip rotor model. (a) The reference blade, (b) The slotted-Tip blade, (c) Local sketch of four circular slots, (d) The right view of blade tip. 
Based on this model, other simulation parameters are consistent with the hovering state (hovering height is $0.52 \mathrm{R}$, blade tip Mach number is 0.63 , increased particle number is 5000 per second). The simulation results of the two types are compared and analyzed.

It can be seen from the Figure 21 that after the time of $t=1.75 \mathrm{~s}$, the average height of particles in the flow field affected by the blade slotting is lower than that of the normal blade, and gradually lags behind that of the normal blade. This situation can show that after the blade slotting, the sand particles in the sand-dust environment will reduce the suspension height because of the weakening of the blade tip vortex, reduce the impact of suspended dust on flight safety, and achieve the effect of restraining brownout.

In order to further analyze the comparison of particle distribution in the air, the results of particle numbers in the height range of $600 \mathrm{~mm}-4000 \mathrm{~mm}$ at different times are compared.

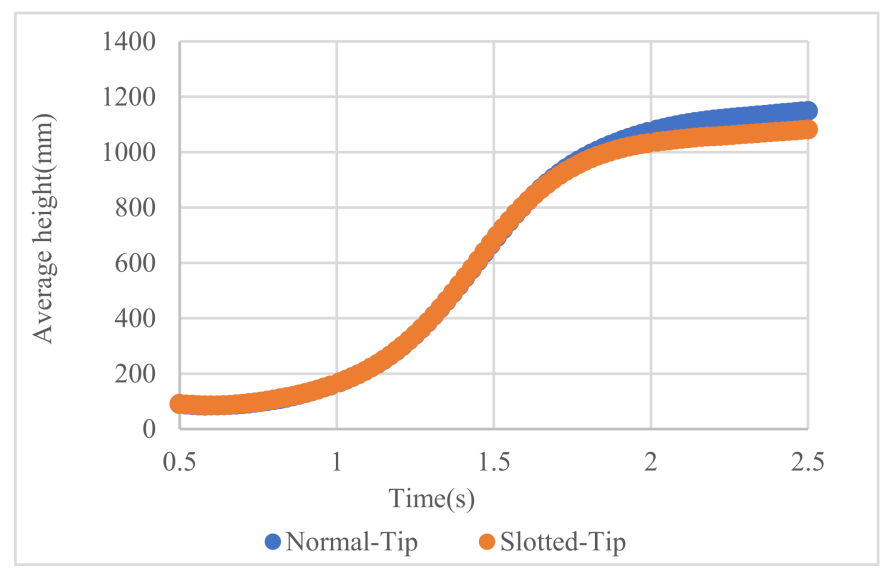

Figure 21. Comparison of average height of particles in different blades by time.

It can be seen from the Figure 22 that the number of particles in the normal blade group will be less than that in the slotted blade group when the height above the ground is low, and the number of suspended particles in the normal blade group will be more than that in the slotted blade group when the distance is more than $3000 \mathrm{~mm}$. From the perspective of helicopter flight safety, the slotted blade can reduce the average height of particle suspension and the reduction of the number of suspended particles near the blade area (the blade height is $4250 \mathrm{~mm}$ ) is more significant, which can effectively reduce the impact of brownout.

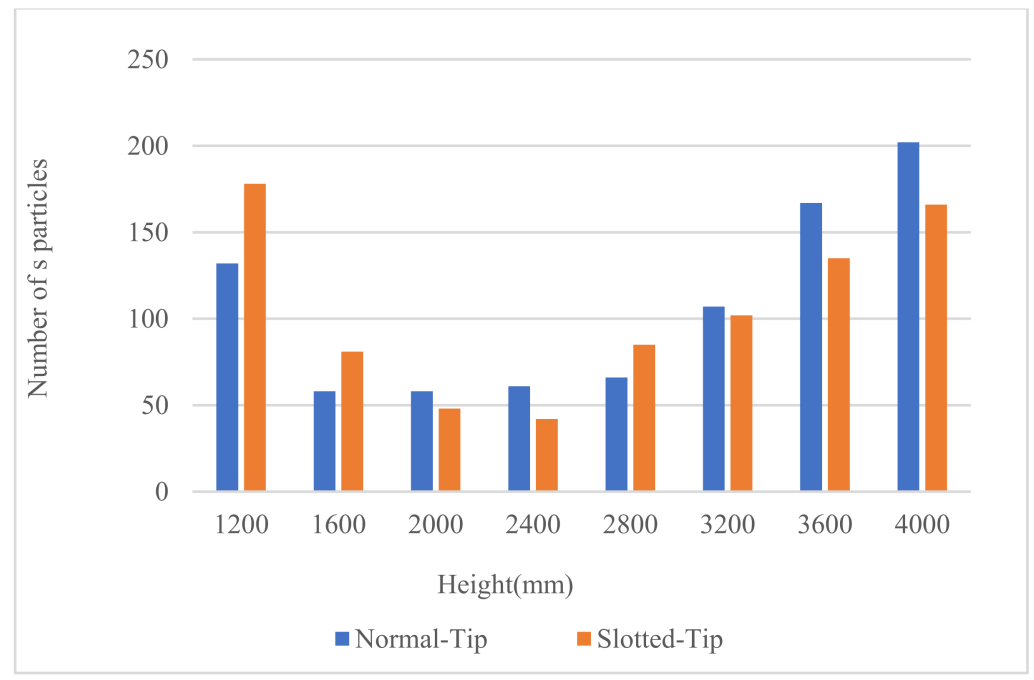

Figure 22. Comparison of particles numbers by height in different blades at $2 \mathrm{~s}$ and $2.25 \mathrm{~s}$. 


\section{Discussion}

Studying the influencing factors of the brownout phenomenon, it is found that hovering height and the size of dust particles directly affect the formation of the brownout phenomenon. These studies can help pilots to adjust their hovering strategies in relevant environments. In addition, through the analysis of particle flight speed and suspension height can help to study the erosion effect of dust particles on blades, which is also a key problem related to brownout. This article considers the hovering state, and can further study the brownout caused by the helicopter landing or take-off process.

\section{Conclusions}

Sand and dust will be entrained into the air under the influence of a helicopter flow field, causing the brownout phenomenon and presenting a serious hidden danger to flight safety. The brownout phenomenon of a helicopter in a sand-dust environment is numerically simulated, and the movement and distribution of sand at different hovering heights are analyzed.

(1) In the brownout phenomenon, the generation of dusty clouds and the entrainment of sand particles are directly related to the ground effect of the rotor. The main reason for all kinds of phenomena is the entrainment of dust from the ground after the blade tip vortex falls off a blade to the ground.

(2) In the sand-dust environment, when the UH60 hovering height is $0.52 \mathrm{R}$, the phenomenon of brownout will occur, and the pilot's field of vision will be covered by the dusty cloud, which presents a serious hidden danger to flight safety. When the hovering height is $1 \mathrm{R}$, the phenomenon of brownout is not obvious. For other helicopters, this value will be slightly different.

(3) The sand particles under the middle of the blade disk will be squeezed by the downwash flow field and move upward, then many particles will contact and collide with the fuselage.

(4) In the brownout phenomenon, most of the sand particles still gather close to the ground. The average velocity of the particles is about $12 \mathrm{~m} / \mathrm{s}$, the height of dusty cloud from the ground will gradually increase over time, and will then remain at a certain height. The radial range of dust will keep expanding for a longer time.

(5) In sand-dust environment, the particles with large radius are less susceptible to upwash than these with small radius. This paper verifies that the particles with a 50 um radius basically are left on the ground, while the particles with $10 \mathrm{um}$ radius will be suspended in the air easily.

(6) Slotting on the swept rotor blade tip can influence on rotor wake, and then reduce the damage of brownout.

Author Contributions: Conceptualization, Y.C.; Data curation, G.W.; Formal analysis, Y.C. and C.J.; Investigation, C.J.; Methodology, G.W.; Project administration, C.J.; Resources, C.J.; Supervision, Y.C.; Validation, G.W.; Writing_original draft, G.W.; Writing—review \& editing, Y.C. All authors have read and agreed to the published version of the manuscript.

Funding: This research received no external funding.

Conflicts of Interest: The authors declare no conflict of interest.

\section{References}

1. Bagnold, R.A. The Physics of Blown Sand and Desert Dunes Methuen; Springer: Dordrecht, The Netherlands, 1941. [CrossRef]

2. Wachspress, D.A.; Whitehouse, G.R.; Keller, J.D.; Yu, K.; Gilmore, P.; Dorsett, M.; McClur, K. A high fidelity brownout model for real-time flight simulations and trainers. In Proceedings of the American Helicopter Society 65th Annual Forum, Grapevine, TX, USA, 27-29 May 2009; pp. 27-29.

3. Sutherland, A.J. Proposed mechanism for sediment entrainment by turbulent flows. J. Geophys. Res. 1967, 72, 6183-6194. [CrossRef]

4. Leese, G.W. Helicopter Downwash Blast Effects Study; US Army Engineer Waterways Experiment Station: Vicksburg, MS, USA, 1964. [CrossRef] 
5. Rodgers, S.J. Evaluation of the Dusty Cloud Generated by Helicopter Rotor Downwash; Msa Research Corp: Evans City, PA, USA, 1968.

6. Leishman, J. Investigation of Sediment Entrainment in Brownout Using High-Speed Particle Image Velocimetry. In Proceedings of the 65th Annual American Helicopter Society Forum, Grapevine, TX, USA, 27-29 May 2009.

7. Lee, T.E.; Leishman, J.G.; Ramasamy, M. Fluid Dynamics of Interacting Blade Tip Vortices with a Ground Plane. J. Am. Helicopter Soc. 2010, 55, 22005. [CrossRef]

8. Nathan, N.D.; Green, R.B. The flow around a model helicopter main rotor in ground effect. Exp. Fluids 2012, 52, 151-166. [CrossRef]

9. Ramasamy, M.; Leishman, J.G. The interdependence of straining and viscous diffusion effects on vorticity in rotor flow fields. In Proceedings of the Annual Forum Proceedings-American Helicopter Society, Phoenix, AZ, USA, 6-8 May 2003; American Helicopter Society, Inc.: Alexandria, VA, USA, 2003; Volume 59, pp. 1787-1801.

10. Syal, M.; Govindarajan, B.; Leishman, J.G. Mesoscale sediment tracking methodology to analyze brownout cloud developments. In Proceedings of the American Helicopter Society, 66th Annual Forum, Phoenix, AZ, USA, 11-13 May 2010; Volume 1, p. 3.

11. Milluzzo, J.I. Effects of Blade Tip Shape on Rotor In-Ground-Effect Aerodynamics; University of Maryland: College Park, MD, USA, 2012; pp. 39-83.

12. Polzin, J.W.; Guntupalli, K.; Rajagopalan, R.G. Discrete Blade Model for Rotorcraft Brownout. In Proceedings of the 29th AIAA Applied Aerodynamics Conference, Honolulu, HI, USA, 27-30 June 2011. [CrossRef]

13. Hance, B.T. Effects of Body Shapes on Rotor In-Ground-Effect Aerodynamics; University of Maryland: College Park, MD, USA, 2012; pp. 42-66.

14. Whitehouse, G.R.; Wachspress, D.A.; Quackenbush, T.R.; Keller, J.D. Exploring Aerodynamic Methods for Mitigating Brownout. In Proceedings of the American Helicopter Society 65th Annual Forum, Grapevine, TX, USA, 27-29 May 2009.

15. Whitehouse, G.R.; Wachspress D A and Quackenbush, T.R. Aerodynamic Design of Helicopter Rotors for Reduced Brownout. In Proceedings of the International Powered Lift Conference, Philadelphia, PA, USA, 5-7 October 2010.

16. Fan, L.S.; Zhu, C. Principles of Gas-Solid Flows; Cambridge University Press: Cambridge, UK, 1999; pp. 164-205.

17. Light, J.S. Tip Vortex Geometry of a Hovering Helicopter Rotor in Ground Effect. J. Am. Helicopter Soc. 1993, 38, 34-42. [CrossRef]

18. Caradonna, F.X.; Tung, C. Experimental and analytical studies of a model helicopter rotor in hover. In Proceedings of the European Rotorcraft and Powered Lift Aircraft Forum, Moffett Field, CA, USA, 8-11 September 1981.

19. Caradonna, F.X.; Laub, G.H.; Tung, C. An Experimental Investigation of the Parallel Blade-Vortex Interaction; Ames Reasearch Center: Moffett Field, CA, USA, 1984.

20. Ahmad, J.U.; Strawn, R.C. Hovering rotor and wake calculations with an overset-grid Navier-Stokes solver. In Proceedings of the 55th AHS International Annual Forum, Montreal, QC, Canada, 25-29 May 1999; pp. 1949-1959.

21. Yu, H.; Cheng, W.; Wu, L.; Wang, H.; Xie, Y. Mechanisms of dust diffuse pollution under forced-exhaust ventilation in fullymechanized excavation faces by CFD-DEM. Powder Technol. 2017, 317, 31-47. [CrossRef] 